\title{
CONTOUR DYNAMICS METHODS
}

\author{
D. I. Pullin
}

Department of Mechanical Engineering, The University of Queensland, St Lucia, Queensland 4072, Australia

KEY WORDS: vorticity, vortex dynamics

\section{INTRODUCTION}

In an early paper on the stability of fluid layers with uniform vorticity $\omega$, Rayleigh (1880) remarks

... In such cases, the velocity curve is composed of portions of straight lines which meet each other at finite angles. This state of things may be supposed to be slightly disturbed by bending the surfaces of transition, and the determination of the subsequent motion depends upon that of the form of these surfaces. For $\omega$ retains its constant value throughout each layer unchanged in the absence of friction, and by a well-known theorem the whole motion depends upon $\omega$.

We can now recognize this as essentially the principal of contour dynamics (CD), where $\omega$ is the uniform vorticity. The theorem referred to is the Biot-Savart law. Nearly a century later Zabusky et al (1979) presented numerical CD calculations of nonlinear vortex patch evolution. Subsequently, owing to its compact form conferring a deceptive simplicity, CD has become a widely used method for the investigation of two-dimensional rotational flow of an incompressible inviscid fluid. The aim of this article is to survey the development, technical details, and vortex-dynamic applications of the CD method in an effort to assess its impact on our understanding of the mechanics of rotational flow in two dimensions at ultrahigh Reynolds numbers.

The study of the dynamics of two- and three-dimensional vortex mechanics by computational methods has been an active research area for more than two decades. Quite apart from many practical applications in the acrodynamics of separated flows, the theoretical and numerical study of 
vortices in incompressible fluids has been stimulated by the idea that turbulent fluid motion may be viewed as comprising ensembles of more or less coherent laminar vortex structures that interact via relatively simple dynamics and by the appeal of the vorticity equation, which does not contain the fluid pressure. Two-dimensional vortex interactions have been perceived as supposedly relevant to the origins of coherent structures observed experimentally in mixing layers, jets, and wakes, and for models of large-scale atmospheric and oceanic turbulence. Interest has often focused on the limit of infinite Reynolds number, where in the absence of boundaries, the inviscid Euler equations are assumed to properly describe the flow dynamics. The numerous surveys of progress in the study of vorticity and the use of numerical methods applied to vortex mechanics include articles by Saffman \& Baker (1979) and Saffman (1981) on inviscid vortex interactions and Aref (1983) on two-dimensional flows. Numerical methods have been surveyed by Chorin (1980), and Leonard $(1980,1985)$. Caflisch (1988) describes work on the mathematical aspects of the subject. Zabusky (1981), Aref (1983), and Melander et al (1987b) discuss various aspects of CD. The review of Dritschel (1989) gives emphasis to numerical issues in $\mathrm{CD}$ and to recent computations with contour surgery.

This article is confined to a discussion of vortices on a two-dimensional surface. We generally follow Saffman \& Baker (1979) in matters of definition. In two dimensions a vortex sheet is a line of discontinuity in velocity while a vortex jump is a line of discontinuity in vorticity. We shall, however, use filament to denote a two-dimensional ribbon of vorticity surrounded by fluid with vorticity of different magnitude (which may be zero), rather than the more usual three-dimensional idea of a vortex tube. The ambiguity is unfortunate but is already in the literature. Additionally, a vortex patch is a finite, singly connected area of uniform vorticity while a vortex strip is an infinite strip of uniform vorticity with finite thickness, or equivalently, an infinite filament. Contour Dynamics will refer to the numerical solution of initial value problems for piecewise constant vorticity distributions by the Lagrangian method of calculating the evolution of the vorticity jumps. Such flows are often related to corresponding solutions of the Euler equations that are steady in some translating or rotating frame of reference. These solutions will be called vortex equilibria, and the numerical technique for computing their shapes based on $\mathrm{CD}$ is often referred to as contour statics.

The mathematical foundation for the study of vorticity was laid primarily by the well-known investigations of Helmholtz, Kelvin, J. J. Thomson, Love, and others. In our century, efforts to produce numerical simulations of flows governed by the Euler equations have utilized a variety of Eulerian, Lagrangian, and hybrid methods. Among the former are the 
class of spectral methods that now comprise the prevailing tool for largescale two- and three-dimensional calculations (see Hussaini \& Zang 1987). The Lagrangian methods for two-dimensional flows have been predominantly vortex tracking techniques based on the Helmholtz vorticity laws. The first initial value calculations were those of Rosenhead (1931) and Westwater (1935) who attempted to calculate vortex sheet evolution by the motion of $O(10)$ point vortices. Subsequent efforts by Moore (1974) (see also Moore 1983, 1985) and others to produce more refined computations for vortex sheets have failed for reasons related to the tendency for initially smooth vortex sheet data to produce singularities (Moore 1979). Discrete vortex methods used to study the nonlinear dynamics of vortex patches and layers have included the evolution of assemblies of point vortices by direct summation (e.g. Acton 1976) and the cloud in cell method (Roberts \& Christiansen 1972, Christiansen \& Zabusky 1973, Aref \& Siggia 1980, 1981). For reviews see Leonard (1980) and Aref (1983). These techniques have often been criticized for their lack of accuracy and numerical convergence and because they may be subject to grid scale dispersion. However, many qualitative vortex phenomena observed in nature and in experiments, such as amalgamation events and others still under active investigation (e.g. filamentation) were first simulated numerically with discrete vortices. The contour dynamics approach is attractive because it appears to allow direct access, at least for small times, to the inviscid dynamics for vorticity distributions smoother than those of either point vortices or vortex sheets, while at the same time enabling the mapping of the two-dimensional Euler equations to a one-dimensional Lagrangian form.

In Section 2 we discuss the formulation and numerical implementation of contour dynamics for the Euler equations in two dimensions. Section 3 is concerned with applications to isolated and multiple vortex systems and to vortex layers. An attempt is made to relate this work to calculations of the relevant vortex equilibria and to results obtained with other methods. Axisymmetric contour dynamics and the treatment of the multi-layer model of quasigeostrophic flows are described in Section 4 while Section 5 is devoted to a discussion of the tendency shown by vorticity jumps to undergo the strange and subtle phenomenon of filamentation.

\section{CONTOUR DYNAMICS}

The first numerical simulations of the unsteady Euler equations using contour dynamics were reported by Deem \& Zabusky (1978) and Zabusky et al (1979). Contour dynamics is related to the "water bag" method for calculating phase space evolution problems of plasma physics and one- 


\section{PULLIN}

dimensional gravitational systems (dePackh 1962, Berk \& Roberts 1970). Roberts \& Christiansen (1972) and Berk et al (1970) discuss the analogy that exists between the two-dimensional vorticity equation for an inviscid fluid and the Vlasov equation of charged particle physics: $\omega(x, y, t)$ is replaced by the one-dimensional phase space distribution function $f(p$, $q, t)$-a function of the phase space conjugate coordinate and momentum $(q, p)$-while the stream function $\psi(\omega)$ is replaced by the Hamiltonian $H(f)$. The analogy is incomplete since vorticity dynamics requires solution of a two-dimensional rather than a one-dimensional Poisson equation as for the case of phase space hydrodynamics. The striking similarities between qualitative features in the phase-plane evolution of plasma and gravitating mass systems and two-dimensional vortex systems (Berk et al 1970), however, clearly points to their common base in the dynamics of Hamiltonian systems. It is well-known that the two-dimensional Euler equations for a constant density fluid are identical to those governing a magnetically confined electron column (Levy \& Hockney 1968), where the charge density $n(x, y, t)$ plays the role of the vorticity $\omega(x, y, t)$. Experiments performed on confined plasmas (e.g. Driscoll \& Fine 1990) are thus relevant to Euler flows.

The Euler equations for two-dimensional flow of an inviscid barotropic fluid $[p=p(\rho), \rho$ being the density] are

$$
\frac{D}{D t}\left(\frac{\omega}{\rho}\right)=0, \quad \omega=\nabla \times \mathbf{u}, \quad \frac{D \rho}{D t}+\rho \nabla \cdot \mathbf{u}=0,
$$

where in three-dimensional Cartesian coordinates $(x, y, z), \mathbf{u}=(u, v, 0)$ is the velocity and $\omega=(0,0, \omega)$ the vorticity. If the fluid is incompressible then $\omega$ is conserved on particle paths, and $\omega$ and $\psi$ are related by the Poisson equation $\nabla^{2} \psi=-\omega$, where $\mathbf{u}=(\partial \psi / \partial y,-\partial \psi / \partial x)$. Contour dynamics follows when $\omega(x, y, 0)$ is piecewise constant with values $\omega_{k}$, $k=1, \ldots K$ in regions $\mathscr{R}_{k}$ bounded by curves (contours) $\mathscr{C}_{k}$ that are vortex jumps. The first of (1) then guarantees that this distribution is maintained by the evolution so that the area integrals resulting from inversion of the Poisson equation can be expressed as line integrals on the $\mathscr{C}_{k}$, which remain material curves. Since the resulting expression for $\mathbf{u}$ is valid for any $\mathbf{r}$, then letting $\mathbf{r}$ approach $\mathbf{r}_{k}$ on $\mathscr{C}_{k}$ and identifying $\mathbf{u}\left(\mathbf{r}_{k}\right)$ with the velocity of the material particle $\mathbf{r}_{k}(e, t)$ (where $e$ is a Lagrangian parameter on $\mathscr{C}_{k}$ ) gives the CD equation (Zabusky et al 1979):

$$
\frac{d \mathbf{r}_{k}}{d t}=-\frac{1}{2 \pi} \sum_{j} \Delta \omega_{j} \oint_{\mathscr{C}_{j}} G\left(\mathbf{r}_{k}, \mathbf{r}_{j}^{\prime}\right) \frac{\partial \mathbf{r}_{j}^{\prime}}{\partial e_{j}^{\prime}} d e_{j}^{\prime}+\mathbf{u}_{\mathrm{ex}}, \quad k=1, \ldots K,
$$

where $\mathbf{u}_{c x}$ is an external velocity field and $\Delta \omega_{k}=\omega_{k}-\omega_{k-1}$. If the $\mathscr{C}_{k}$ are 
closed curves (not necessarily nested) and there are no solid boundaries then $G=\log \left|\mathbf{r}_{k}-\mathbf{r}_{j}^{\prime}\right|$. Dritschel (1989) gives forms of the Greens function $G$ for various boundary conditions including streamwise periodic vortex strip distributions. Equations (2) are a set of closed evolution equations for $\mathbf{r}_{k}\left(e_{k}, t\right)$. Their solution is a singular solution of the incompressible Euler equations in two dimensions. For closed $\mathscr{C}_{k}$ in an unbounded fluid, there are several invariants of the evolution: the areas of the $\mathscr{R}_{k}$, the linear impulse $X$, the angular impulse $J$, and the excess energy $E$. Expressions for these quantities in terms of integrals on the $\mathscr{C}_{k}$ are readily obtained (see Dritschel 1989). Several different versions of CD have been used including the use of complex variables (e.g. Pullin 1981, Baker \& Shelley 1990).

Contour dynamics may be also obtained directly from the equation

$$
\nabla^{2} \mathbf{u}=\nabla(\nabla \cdot \mathbf{u})-\nabla \times \boldsymbol{\omega}
$$

Equation (3) shows that contour dynamics for an incompressible fluid requires initial distributions $\omega(\mathbf{r}, 0)$ such that the vorticity dynamics confines the support of $\nabla \times \omega$ to surfaces whose shape is independent of the coordinate parallel to $\omega$. Such constraints can be found for example in Cartesian, cylindrical, and helical coordinates [the last example has been suggested by G. R. Baker and M. J. Shelley (personal communication)]. Leonard (1985) points out that because the transport equation for $\nabla \times \omega$ generally contains source terms in three dimensions, there is no threedimensional equivalent of contour dynamics. From (1) and (3) it follows that CD also fails for compressible two-dimensional flow because $\omega / \rho$ is the conserved quantity and the velocity is not solenoidal.

The equations of contour statics describing vortex equilibria with piecewise constant vorticity are obtained by first interpreting the right-hand side of (2) as the Eulerian velocity field $\mathbf{u}(x, y, t)$. The boundary condition $\mathbf{u} \times \partial \mathbf{r}_{k}(e) / \partial e=0$ applied on the $\mathscr{C}_{k}$ in an appropriate translating or rotating frame of reference-defined by a suitable choice of $\mathbf{u}_{\mathrm{ex}}$ together with a set of constraint relations fixing continuation parameters - then gives a closed set of nonlinear integral equations for the shapes of the $\mathscr{C}_{k}$. These can be solved by standard numerical methods. Bifurcations to new solution branches can be found by locating points of neutral linear stability (e.g. Kamm 1987).

\section{Numerical Implementation}

A numerical solution of (1) requires a representation of $\mathscr{C}$ (we omit the subscript for convenience), a means of accurate evaluation of the line integrals, and a method for integrating forward in time. Most workers utilize a discretization of $\mathscr{C}$ into a set of $N(t)$ nodes in the $(x, y)$ plane 


\section{PULLIN}

where we note the dependence of $N$ with time. The curve $\mathscr{C}$ is then defined by a set of linear or higher order elements on the node set. For evaluation of the integral it is generally beneficial to remove the singularity through integration by parts. This gives an integrand of the form $\left[\mathbf{r}-\mathbf{r}\left(e^{\prime}\right)\right] \partial G / \partial e^{\prime}$, which is bounded but not analytic when $\mathbf{r} \rightarrow \mathbf{r}\left(e^{\prime}\right)(\mathbf{r}$ not on $\mathscr{C})$. This nonanalyticity can produce a "close approach problem" associated with rapid variation of the integrand when two contours or portions of the same contour become nearly parallel ( $\mathbf{r}$ is on one contour and $\mathbf{r}^{\prime}$ on the other), with a minimum distance on the order of the local node spacing. When $G=\log \left|\mathbf{r}-\mathbf{r}^{\prime}\right|$, this problem does not arise if linear (e.g. Zabusky et al 1979, Overman \& Zabusky 1983) or quadratic elements (Jacobs \& Pullin 1989) are used, since their contributions to the line integral can be evaluated analytically. When $\mathscr{C}$ is an open curve with spatially periodic shape, closed form integration is not possible even for linear elements and the close approach problem requires special care. Various methods have been used including use of high order interpolation (Baker \& Shelley 1990), extraction and closed form integration of the nonanalytic part of the integrand (Jacobs \& Pullin 1989), and the use of circular arc approximations (Pozrikidis \& Higdon 1985). Dritschel (1988b, 1989) uses a scheme suitable for open or closed $\mathscr{G}$ consisting of cubic contour elements combined with expansion of the integrand. For all these schemes evaluation of the velocity field on the complete node set is an $O\left(N^{2}\right)$ operation. Reduction to $O(N \log N)$ by use of moment or multipole expansions for remote contours has yet to be reported.

The use of node adjustment schemes wherein $N(t)$ is generally a monotonically increasing function has been necessary for CD calculations, even to small times, owing to the material deformation of the contours in which near exponential growth in the contour perimeter is accompanied by the rapid production of fine scales. These schemes have been generally based on the deletion or insertion of nodes locally in order to achieve a distribution in which the local node spacing lies within prescribed bounds that depend on contour geometry, including curvature and the proximity of other contours. Each group of workers has developed its own node control scheme [but see Zabusky \& Overman (1983) and Zou et al (1988) for a discussion of optimum node distribution], usually implemented every few integration time steps $\Delta t$. The reader is referred to the various cited papers. Dritschel (1988b) uses a scheme that is nonlocal in the sense that the node density depends on the velocity. Complete node redistribution based on the cubic contour elements used for the linc integrals is implemented at each $\Delta t$. This appears to give substantially smoother contour behavior when compared with local node adjustment methods at the price of loss of the initial node set. 
The discretized CD equations are a set of $2 N$ first order ODEs, which cumulative experience indicates are benign in the sense of nonstiffness. Baker (1990) nevertheless finds a spurious but slow instability for circular vortices arising from discretization. Fourth order Runge-Kutta or other standard ODE integrators are suitable, but see Section 5 on symplectic integration methods. Various tests of accuracy have included comparison with analytic solutions (Moore \& Saffman 1971, Kida 1981) for elliptical vortices, monitoring the conserved integrals (most notably $E$ ), convergence studies of standard vortex merger problems using sequences of increasingly dense node sets, and comparison with spectral methods (see Dritschel 1989). Specific calculations may be either dissipative or antidissipative (i.e. $E$ may actually increase) and in so far as $E$ is a functional of $\mathbf{r}(e)$ [a double integral: see Equation (14)], the numerical dissipation properties of CD are global.

\section{Contour Surgery}

In response to obstacles presented by the production of fine scales in standard CD, Dritschel (1988b, 1989) developed the method of contour surgery (CS). In this variant of CD the algorithm automatically removes scales of the flow smaller than a predefined lower limit, say $\delta$. There are two principal features of the scheme. First, when two contours enclosing identical vorticity approach nearer than $\delta$, they merge into a single contour. Conversely a single contour may divide in two when the distance between two distinct portions decreases below $\delta$. Second, an artificial angle or corner is forced whenever the local curvature exceeds $\delta^{-1}$. Reversion of corners to simple nodes is also possible. By this means repeated folding of contours with the associated explosion in $N(t)$ is reduced. Dritschel (1989) claims with supporting examples that CS calculations showing five orders of magnitude in scale separation are possible with $N$ of order $10^{4}$. Figure 1 shows a CS calculation with $\delta=2 \times 10^{-4}$. Contour surgery is required after a relatively short time, underlining the rapid enstrophy cascade to very fine scales. The preservation of integral invariants is comparable to standard CD. Dritschel states that the complexities of CS present a "logical nightmare" comprising a thousand lines of code, and Dritschel (1988b) contains an interesting chapter titled "blind alleys."

Contour surgery is not for the numerically faint hearted. While a simple $\mathrm{CD}$ algorithm without detailed node control presents a straightforward programming exercise, refined codes incorporating CS appear more logically complicated than their finite difference or spectral counterparts. A price is paid for reducing the effective dimensionality of the problem by one. 
A Annual Reviews

www.annualreviews.org/aronline

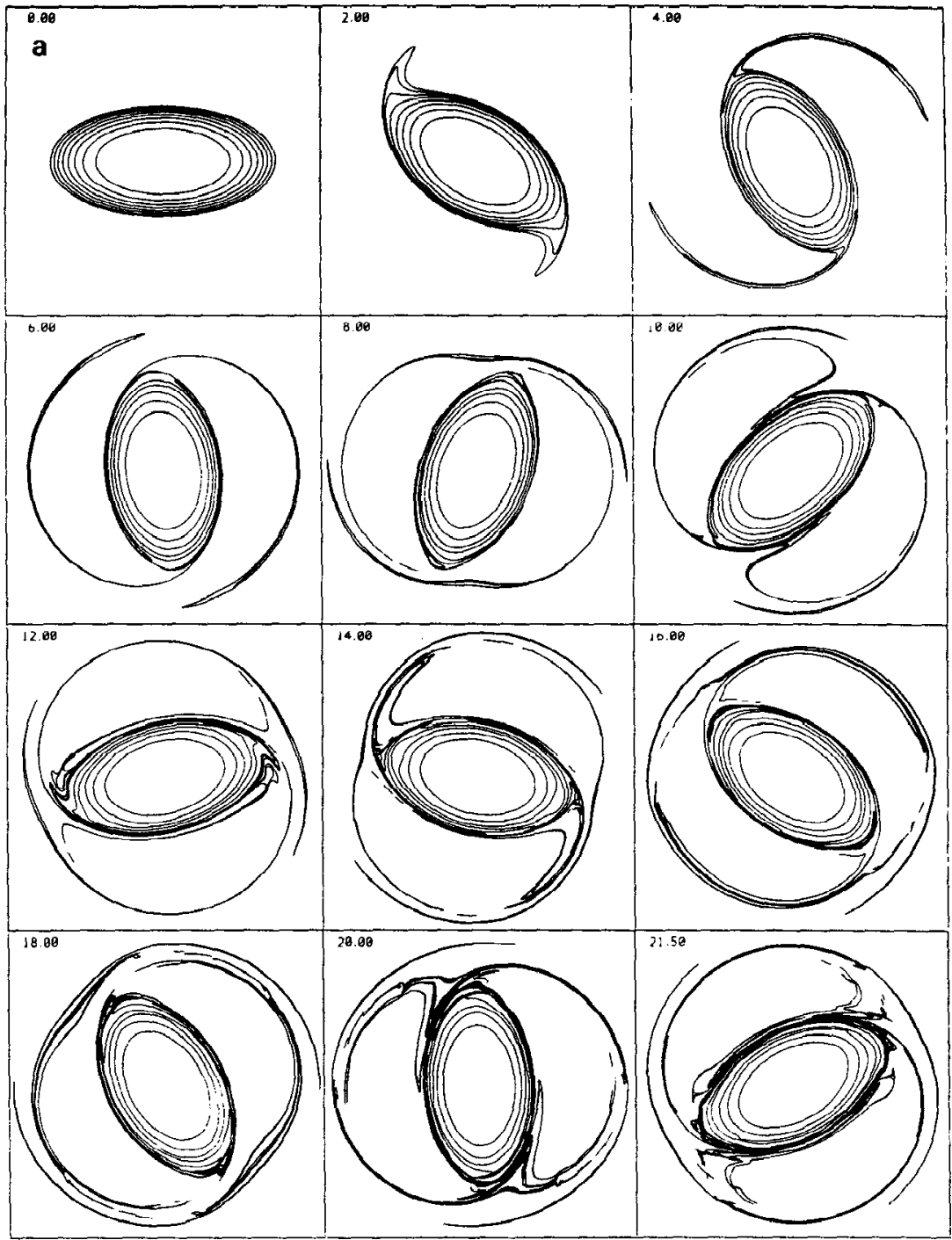

Figure 1 The evolution of an elliptical distribution of vorticity computed with contour surgery. At $t=21.5$ there are a total of 16,128 nodes. [From Dritschel (1989). Printed with permission.] 


\section{TWO-DIMENSIONAL VORTICES IN AN INVISCID FLUID}

The study of interactions between vortices in two dimensions received stimulus in the 1970s from the coherent structure hypothesis for the largescale dynamics of the formation stages of wakes, mixing layers, and jets. [See Cantwell (1989) for a contemporary account of the controversy that still surrounds this subject.] While even such simple flows exhibit threedimensional features, two-dimensional Navier-Stokes or Euler vortex models have successfully reproduced the qualitative behavior of large scales. We further note than one of the few dynamical predictions of the Kolmogorov $k^{-5 / 3}$ inertial range energy spectrum for homogeneous turbulence (Lundgren 1982) uses a quasi-two-dimensional vortex model of fine scales. Two-dimensional simulations of temporal shear layers and spatially evolving mixing layers (sec Aref 1983 for a survey) typically show roll-up of the layer into finite vortex cores, which then interact through higher order instabilities --in qualitative agreement with experiments such as those of Winnant \& Browand (1974). The subsequent interest in the dynamics of isolated vortices with the effects of surrounding vorticity often modeled by an imposed strain received further impetus from growing interest in the predominantly two-dimensional large scales seen in geophysical flows. Related spectral calculations of two-dimensional turbulence (e.g. Fornberg 1977, McWilliams 1984, Henshaw et al 1989) show the spontaneous formation of finite vortex cores from random periodic initial vorticity distributions.

\section{Isolated Vortex Cores}

Vortex patch equilibria have been widely used to study the dynamics of finite vortices. Saffman $(1982,1988)$ has reviewed the extensive literature on the subject. The circular Rankine patch of vorticity $\omega$ surrounded by irrotational fluid supports steadily rotating infinitesimal disturbances to its perimeter shape of the form $\exp [i(m \theta-\sigma t)]$, where $\theta$ is the angular coordinate and $m$ the azimuthal wavenumber. Bifurcations from the circular vortex patch occur when $\sigma=0$, and continuation to finite amplitude disturbances gives the noncircular rotating vortex equilibria with $m$-fold symmetry. Thus $m=2$ gives the rotating Kirchoff ellipsc, while $m=3,4 \ldots$ gives the so called rotating V-states, the first few of which were calculated using contour statics by Deem \& Zabusky (1978) (see also Su 1979). The stability of these states can be calculated in turn and further bifurcations appear, some to nonsymmetrical shapes (Kamm 1987). This leads Saffman (1988) to conjecture the existence of an infinite set of steadily rotating vortex patch solutions for given patch area. One can expect similar non- 
uniqueness for finite area isolated vortices with piecewise constant vorticity distributions (Polvani \& Flierl 1986, Dritschel 1989) or for multiply connected patches (Dritschel 1986).

Contour dynamics has been used extensively to calculate the nonlinear stability of small but finite amplitude disturbances to various classes of both linearly stable and unstable steadily rotating vortex patches (Deem \& Zebusky 1978, Zabusky 1981, Overman \& Zabusky 1983, Wu et al 1984, Dritschel 1986, 1989). In view of the probable infinite multiplicity of steady equilibria one seeks general results, one of which is that $L^{2}$ stability is just conservation of angular momentum, while $L^{1}$ stability follows from the Schwartz inequality combined with area conservation (Saffman 1985; see also Dritschel 1988a). The picture emerging from the numerics shows the boundaries (in the spaces of the relevant solution branches) of linear and nonlinear stability to be broadly similar. Growth following linear instability leads to the formation of increasingly complex and progressively finer patterns of either intrusive or of extrusive filaments of vorticity being drawn off the main vortex and wrapping around the core in nearly concentric circles (see Dritschel 1989 for examples). For short times the linearly stable states remain intact (e.g. Overman \& Zabusky 1983) but exhibit perimeter oscillations. Dritschel (1988c), however, presents numerical evidence (discussed in Section 5) that suggests that even stable states are subject to long time filamentation following arbitrarily small disturbances.

These results raise questions concerning the possible existence of asymptotic equilibrium states corresponding to an isolated continuous or piecewise continuous initial distribution of vorticity in an inviscid fluid. Melander et al (1987a) use a pseudospectral calculation with growth of subgrid scales (subgrid filamentation?) damped by a 4th-order hyperviscosity to demonstrate the relaxation to near axisymmetry of an almost uniform elliptical vorticity distribution. They suggest "axisymmetrization", driven by filamentation and occurring on a time scale $D^{2} / \Gamma$, as a general equilibration principle. Dritschel (1989) and Legras \& Dritschel (1990), however, show counterexamples, one of which is reproduced in Figure 1. A CS simulation of an elliptical vortex with piecewise constant vorticity in eight regions appears to show stabilization of the elliptic core surrounded by filament debris. There is no sign of transition to near axisymmetry. Even with CS it was not possible to carry the computation to decisively long times owing to the rapid cascade to fine scales shown in the remarkably complex filament cloud near the core boundary. This result is supported by experiments on magnetically confined electron columns (Driscoll \& Fine 1990) where a structure very like that of Figure 1 is maintained over hundreds of rotation periods.

Long time equilibria are expected to have a statistical character. This is 
because the instantaneous radial vorticity profile resulting from filamentation and subsequent convective mixing will be spiky for initially piccewise constant distributions (Aref 1983 refers to a "jelly roll" structure) and may be asymptotically nondifferentiable even for smooth initial distributions. The statistical equilibria of assemblies of point vortices calculated by Pointin \& Lundgren (1976) may be relevant although Saffman \& Baker (1979) caution against application of results for finite systems to solutions of the Euler equations in the thermodynamic limit. Miller (1990) has developed statistical mechanics for the Euler equations in two dimensions. The theory gives field equations relating the initial vorticity distribution to a coarse-grained asymptotic statistical equilibrium, which is a global extremum of the energy. Only the excess energy and integrals linear in $\omega$ are conserved. It would be interesting to compare solutions of the strongly coupled nonlinear integrodifferential field equations with ensembles of solutions of the Euler equations obtained via $\mathrm{CD}$ or other means.

An extension of the isolated vortex patch is to place the core in a uniform strain field representing the mean motion of surrounding vortices such as other members of a linear array. The basic theoretical result is due to Moore \& Saffman (1971) who found solutions corresponding to elliptical vortex patches of aspect ratio $\theta=b / a$ rotating steadily in a uniform strain field, such that $\theta=f(\beta / \omega, \Omega / \omega)$, where $\beta$ is the strain rate and $\Omega$ the rotation rate. A generalization as given by Kida (1981) who demonstrated that an exactly elliptical patch remains so for all time and may undergo a variety of complex motions with generally time-varying aspect ratio depending on $\beta / \omega$ and $\Omega / \omega$. Neu (1984) added a component of strain that stretches vortex lines. The Moore-Saffman stable and unstable solutions appear as critical points in an appropriate phase plane. Some CD simulations for Neu vortices were given by Jacobs \& Pullin (1985), however Pullin \& Jacobs (1986) show examples in which a strained elliptical patch is a poor model of a vortex with internal structure. Dritschel (1990b) has studied the linear stability of elliptical patches with time dependent aspect ratios using Floquet theory and their nonlinear response to small but finite amplitude disturbances using CD. There are apparently discrepancies in the sense that unstable solutions exhibit a variety of nonlinear phenomenology, some exhibiting partial disintegration by filamentation while others show coarse-grained recurrence of the patch boundary shape.

\section{Multiple Vortices}

Campbell \& Ziff $(1978,1979)$ catalog stable and unstable equilibria of $N$ point vortices up to $N=50$. If all such equilibria can be continued to finite and perhaps different area and uniform vorticity for each vortex, then the class of finite area $N$-vortex equilibria is expected to be very dense, exclud- 
ing even the possibility of further bifurcations off each branch for given $N$. Most work has centered on two cases of $N=2$ equilibria each with two-fold symmetry: the corotating pair and the counter-rotating steadily translating pair. Of these the corotating pair is of interest as a canonical model of two-vortex amalgamation in the mixing layer. The equilibrium shapes were calculated by Saffman \& Szeto (1980) who also used an energy argument of Kelvin to show pair instability for $x_{\mathrm{c}} \leqslant 3.2(A / \pi)^{1 / 2}$ where $x_{\mathrm{c}}$ is the pair centroid separation and $A$ the individual vortex area (see also Dritschel 1985 and Kamm 1987). Overman \& Zabusky (1983) used $\mathrm{CD}$ to calculate the nonlinear evolution of the corotating states finding pair merger for linearly unstable states and perimeter oscillations otherwise. This gives an approximate criterion for merging but one that is limited to vortex patches of shape near the Saffman \& Szeto equilibria. Melander et al (1988) use the second-order moment model of Melander et al (1986) to attempt a more general analysis. They construct a phase plane portrait corresponding to the pair evolution in a set of suitably transformed moment variables from which they identify regions of vortex centroid collapse (merging), corotation without merging, and nutation. This gives a specific set of collapse criteria for general distributions with two-fold symmetry, in terms of the dimensionless impulse $2 \pi J /\left(\omega A^{2}\right)$. CD and pseudospectral calculations of merger play the role of verification experiments. Melander et al (1988) claim that the asymptotic merged vortex state is the axisymmetric monopole. They also give heuristic arguments to suggest that when a small but finite viscosity is present, the outcome of the inviscid interaction is a metastable equilibrium, after which inevitable merger and relaxation presumably towards the Oseen decaying vortex solution occurs.

Jacobs \& Pullin (1985) study the effect on symmetrical two-vortex merger of a three-dimensional straining field with uniform strain rates of the form $(-\beta, \beta-\gamma, \gamma)$, where $\gamma$ is the rate of strain parallel to vortex lines. They claim that this model is relevant to vortex amalgamation in mixing layers when $\gamma=0$ (the strain is provided by neighboring vortices) and to the very large Reynolds number behavior of Corcos-Lin vortices (Lin \& Corcos 1984) when $\gamma>0$ (see also Pullin \& Jacobs 1986). Contour dynamics can still be used because the vorticity of patches is simply amplified uniformly by $\exp (\gamma t)$. Equivalently, the flow can be mapped by a wellknown transformation to a strictly two-dimensional flow with a time dependent strain $(-B, B, 0)$, where $B=(\beta-\gamma / 2) /(1+\gamma t)$.

There are few detailed studies of vortex equilibria or interaction for more than two vortices. Overman \& Zabusky (1982) used CD to study collisions between translating coaxial vortex pairs, observing "leapfrogging" when the vortex areas are small and asymmetrical convective 
merging of like-signed members of each pair otherwise. Dritschel (1985) calculates steady shapes, linear stability, and energetics for families of $N=2-8$ identical corotating vortices. He finds finite area instabilities, which for $N<7$ correspond to boundary distortions and for $N \geq 7$, to modified point-vortex modes. The nonlinear evolution of unstable states calculated with CD (Dritschel 1986) generally indicates merging toward a single structure with large-scale connectivity that may depend on the symmetries of the initial perturbation. The equilibrium shapes and linear stability of colinear and corotating tripole states was studied by Polvani \& Carton (1990), who also calculated the nonlinear evolution of some configurations with a CS algorithm. As mentioned previously the class of multiple-vortex equilibria is very large.

\section{Periodic Vortex Layers and Arrays}

The streamwise periodic temporal shear layer and the double shear layer flow have often been used as approximations to spatially developing mixing layers and wakes respectively. A very simple inviscid model is a single strip of uniform vorticity $\omega$ and thickness $H$ in an otherwise irrotational flow, for which Rayleigh (1880) showed that contour perturbations proportional to $\exp (\sigma t+i k x)$ have maximum growth $\sigma_{\max } \cong 0.2 \omega$ when $k_{\max } H \cong 0.8$, where $k=2 \pi / \lambda$ is the wavenumber and $\lambda$ the wavelength. Disturbances with large $k H$ are stable. Pozrikidis \& Higdon (1985) study the nonlinear response of a uniform strip to a single mode perturbation at near $k_{\max } H$ and observe roll-up into nearly elliptical cores with trailing braids. As the braids roll around the main core they do not exhibit secondary instability. Dritschel (1990a) attributes this behavior, generic to vortex roll-up flows, to stabilization of filaments by the adverse shear felt in the frame of reference that rotates locally with the filament fluid. Wakelike flows may be modeled by contiguous strips containing uniform vorticity of opposite sign. Pozrikidis \& Higdon (1987) find a variety of phenomenology for compound wakes and layers including the spontaneous formation of finitearea vortex pairs of the kind observed in genuinely two-dimensional soap bubble flows (Couder \& Basdevant 1986).

When $H \rightarrow 0$ and $\omega \rightarrow \infty$ such that $\omega H \rightarrow U$, where $U$ is the mean velocity difference across the layer, the thin vortex strip approaches the vortex sheet limit for which the growth rate of a disturbance with wavenumber $k$ becomes $\sigma^{2}=U^{2} k^{2} / 4$. There are analytical (Moore 1979, 1985; see also Caflisch 1988) and numerical (Meiron et al 1982) results that indicate that vortex sheets can develop derivative singularities in a finite time from smooth initial data. The behavior beyond the singularity formation time remains an open question but one that is perhaps the major outstanding problem of vortex sheet mechanics. Baker \& Shelley (1990) 


\section{PULLIN}

investigate the vortex sheet limit of a finite vortex layer by using $C D$ to examine the evolution of a sequence of vortex layers with decreasing but finite $H$ and constant $U$. The numerics indicate that central bulge (core) dimension scales approximately as $(H / \lambda)^{0.775}$, while an estimate of the effective local vortex sheet strength at the bulge diverges as $(H / \lambda)^{-0.225}$. Although very high contour curvature is generated where the braids meet the core, there is no indication of singularity formation in a finite time. Away from the core the trailing braids begin to roll up like a double spiral in qualitative agreement with the desingularized sheet calculations of Krasny (1986), and presumably the evolution obeys the equation for a layer of very small but finite thickness obtained by Moore (1978). The smallest $H / \lambda$ used by Baker and Shelley was constrained by the requirement of adequate spatial resolution (node insertion or redistribution was not used) in the region of maximum contour curvature. Their minimum value, $H / \lambda=0.025$, appears to be too large to resolve the very nonuniform approach to the vortex sheet limit. Computation of the uniform vortexlayer evolution with very small but finite $H / \lambda$ poses a challenging problem. This would be a good test case for the CS method.

Contour dynamics calculations may allow computation of the effective infinite Reynolds number limit for unbounded two-dimensional flows but at the price of replacing continuous vorticity distributions by piecewise constant ones. Most CD calculations use only a single uniform patch or layer: firstly, because the shape and stability of the relevant vortex equilibria can be readily calculated and secondly, because it may be argued that the nonlinear behavior of the system is determined more by the overall vortex structure than by its precise details. This is not always true: The algebraic instability for vortices contained in a circle (Smith \& Rosenbluth 1990) requires a hollow and smooth radial vorticity distribution. An important question is how well a piecewise constant vorticity distribution with multiple contours can model the dynamics of a continuous distribution. Jacobs \& Pullin (1989) utilize an eight-contour and in one case a sixteencontour representation of a Gaussian vorticity distribution across a shear layer. They consider the layer response to perturbations consisting of combinations of eigenfunctions of the linear stability analysis. Tearing, and two- and three-vortex amalgamation can be so stimulated. Figure 2 shows an example of pairing compared with experiment and Navier-Stokes calculation. The initial perturbation for three-vortex merging consists of equal weights of the fundamental mode with maximum linear growth and its second subharmonic, which has a comparable growth rate. Calculations with more subharmonics were not done but the inference may be drawn that multiple vortex interactions would be likely for any reasonable initial distribution of modes. This supports Aref's contention (Aref 1983) that the shear layer should not be viewed as a spatial and temporal periodic 

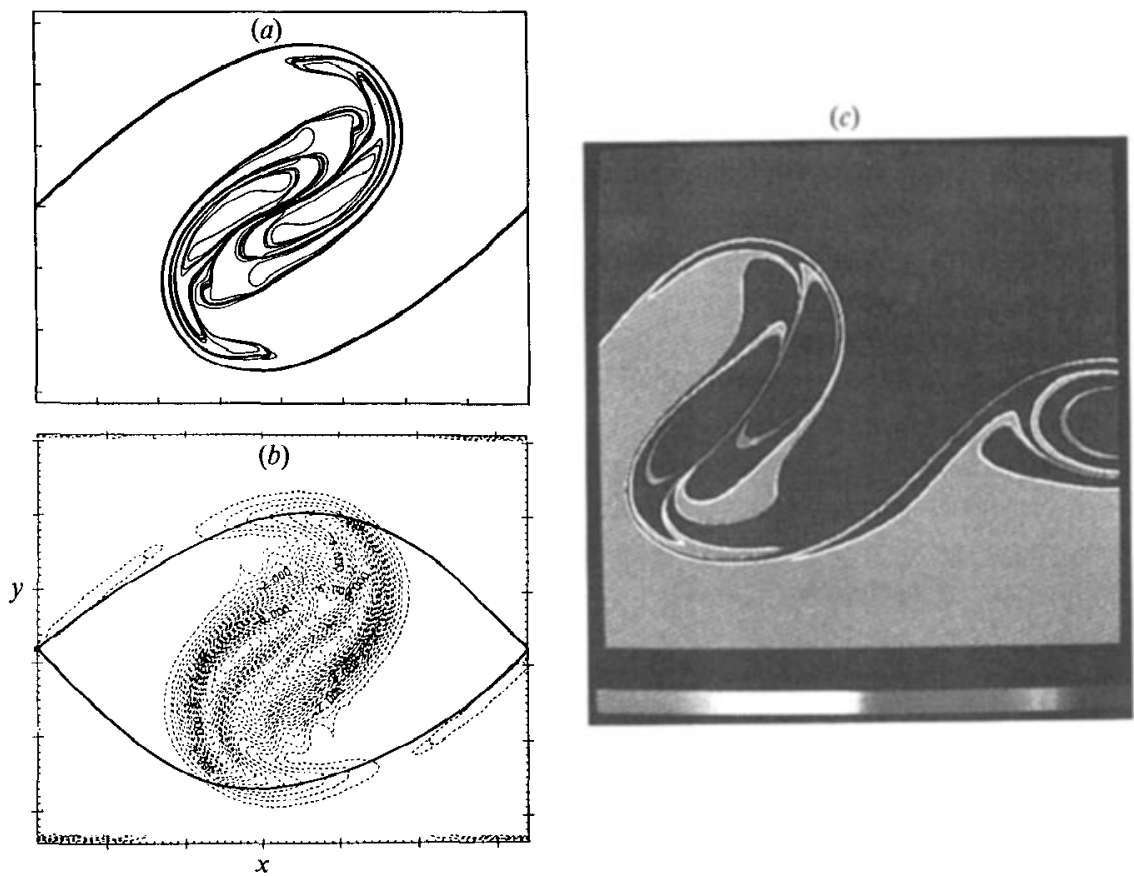

Figure 2 Comparisons of shear layer evolution involving a pairing event: ( $a$ ) CD simulation with 8 contours. (b) Finite difference calculation at $\mathrm{Re}=100$ based on layer thickness (Corcos \& Sherman 1984). (c) Digital laser-induced fluorescence picture of pairing event at $\operatorname{Re}=1750$ (Koochesfahani \& Dimotakis 1986). [From Jacobs \& Pullin (1989). Printed with permission.]

continuation of core formation followed by two-vortex interactions but rather should be seen as a many-body problem in which these events are most probable but not dominant.

The question of approach to a continuous distribution has been studied by Dritschel (1989) using as a test problem the distortion by background shear of a doubly periodic vortex array. He compares the results of an eight contour CS calculation with those from a $1024^{2}$ pseudo-spectral calculation performed with sixth order hyperviscosity. Good agreement at large and medium scales is found but there are differences of detail on the scale of the contour spacing where small kinks appear in the CS results. Similar kinks were also found by Pullin \& Jacobs (1986) in four contour CD calculations of the inviscid dynamics of Corcos-Lin vortex arrays. The kinks appear to be associated with the vorticity jumps in the piecewise constant model. This may be cause for concern in the interpretation of fine-scale contour filamentation exhibited by vortex equilibria, which is discussed in Section 5. 


\section{PULLIN}

Large Reynolds number mixing layer and wake flows containing finite vortex cores have been modeled with single linear arrays (Saffman \& Szeto 1981) and double staggered arrays (Saffman \& Schatzman 1981) of vortex patch equilibria. Saffman (1988) summarizes work on the stability of the single row (Kamm 1987) and the double row, or finite area Karman vortex street (Saffman \& Schatzman 1982, Meiron et al 1984, and others). The nonlinear stability properties of these equilibrium configurations do not seem to have been subject to systematic study using CD.

\section{AXISYMMETRIC AND QUASIGEOSTROPHIC FLOWS}

\section{Axisymmetric Contour Dynamics}

Pozrikidis (1986) and Shariff et al (1989) have constructed contour dynamics methods for inviscid axisymmetric flow. They consider flow without swirl in cylindrical coordinates $(x, r, \theta)$, where $x$ is the axial and $r$ the radial coordinate. The velocity is $\mathbf{u}=(u, v, 0)$ and the vorticity is $\omega=$ $(0,0, \omega)$. The first of Equations (1) is replaced by $D(\omega / r) / D t=0$, so that $\omega / r$ is conserved, reflecting the stretching of circular vortex lines proportional to $r$ that accompanies radial displacement of material particles. Axisymmetric CD follows by first assuming an initially piecewise constant distribution of $\omega / r$, say $\omega(x, r, t)=\lambda_{k} r$ in regions $\mathscr{R}_{k}, k=1 \ldots, K$ of the $(x, r)$ plane, where the $\lambda_{k}$ are constants. The Poisson-like equation relating $\omega$ to the Stokes streamfunction $\psi$ can be inverted to give $\psi$ (Lamb 1932, Section 161) as a sum of area integrals over the $\mathscr{R}_{k}$. These can then be transformed to contour integrals giving equations for $d \mathbf{r}_{k} / d t$ like (2) with $G$ containing complete elliptic integrals. However, the line integrals cannot be evaluated analytically even on straight segments. Shariff et al (1989) use two-point Gaussian quadrature for segments remote from the field point combined with analytical integration of a series expansion for the elliptic integrals otherwise. Pozrikidis uses singularity extraction, which appears to deal with the close contour approach problem.

Moffatt \& Moore (1978) showed by analytical means that the response of a Hills spherical vortex (see Batchelor 1967, p. 526) to an axisymmetric disturbance that distorts the vortex boundary into an oblate/prolate spheroid is to entrain/detrain a narrow spike of irrotational/rotational fluid into/out-of the vortex at the rear stagnation point. This behavior is confirmed by Pozrikidis (1986). He finds that entrainment may wind irrotational fluid about the rotational core giving a nearly steady ring with structure similar to the member of the Norbury-Fraenkel family (Norbury 1972, 1973, Fraenkel 1972) with the same energy and impulse as the initial 
flow. Shariff et al (1989) dispute this, instead finding a high wavenumber sawtooth instability. They also investigate passage interactions and headon collisions between coaxial vortex rings with small cores and with initial boundary shapes modeled by the Norbury-Fraenkel family. Head-on collisions exhibit unexplained behavior in that, as the nearly touching ring pair undergo mutual radial stretching, the adjacent cores evolve through shapes very similar to the Pierrehumbert $(1980,1981)$ two-dimensional pairs, and appear to approach a configuration very like the Sadovskii (1971) limit in which the cores touch.

\section{Quasigeostrophic Contour Dynamics}

Contour dynamics methods may be used for the study of multi-layer rotating fluids that obey the quasigeostrophic equations (see Pedlosky 1987, Chapter 6). Such fluids can support baroclinic instability, which depends on both stratification and rotation and which is of interest in oceanographic and meteorological applications. The continuous vertical density stratification is replaced by horizontal layers, each of constant but slightly different density and of small mean thickness compared with the horizontal scale. If the Rossby number Ro $=U /(2 \Omega L)$ is small (geostrophic balance) (where $U$ is the velocity, $L$ is the length scale, and $\Omega$ is the rotation rate), then the (unfortunately named) potential vorticity $\Pi$ is conserved in each layer. The simplest system is the two-layer model, which is known to support point vortices and their mutual interactions (e.g. Hogg \& Stommel 1985). If $\Pi_{j}=\left(\omega_{j}+2 \Omega\right) / d_{j}$ is the potential vorticity of layer $j$ with local thickness $d_{j}$ and mean thickness $D_{j}(j=1,2)$, then the two-dimensional streamfunctions $\psi_{j}$ are given by

$$
\begin{aligned}
& \Pi_{1}=\nabla^{2} \psi_{1}+\lambda^{2}\left(\psi_{2}-\psi_{1}\right), \\
& \Pi_{2}=\nabla^{2} \psi_{2}+\varepsilon \lambda^{2}\left(\psi_{1}-\psi_{2}\right),
\end{aligned}
$$

where $\lambda^{2}=2 L \Omega\left[\rho /\left(g D_{1} \Delta \rho\right)\right]^{1 / 2}$ is the ratio of $L$ to the radius of deformation (Rossby radius) in the upper layer, $\rho$ and $\rho+\Delta \rho$ are the densities in the upper and lower layers respectively, $g$ is acceleration due to gravity, and $\varepsilon=D_{1} / D_{2}$. If the $\Pi_{j}$ have piecewise constant initial distributions, then inversion of $(5)$ for $\left(\psi_{1}, \psi_{2}\right)$ leads to a $\mathrm{CD}$ formulation for the curves marking the potential vorticity jumps in much the same way as for Equation (2) except that the Green's functions are now more complicated and the right side of (2) must now be summed over the vertical layers.

For the special case when $\Pi_{2}=0$ in the lower layer $G$ takes the form (Polvani et al 1989b)

$$
G(r ; \varepsilon, \lambda)=\frac{\varepsilon}{1+\varepsilon} \log (r)-\frac{1}{1+\varepsilon} K_{0}\left[\lambda(1+\varepsilon)^{1 / 2} r\right],
$$




\section{PULLIN}

where $r=\left|\mathbf{r}-\mathbf{r}^{\prime}\right|$ and $K_{0}$ is the modified Bessel function of order zero. Equation (5) shows $\lambda$ as a coupling constant between layers and when $\lambda=0$, each then obeys the two-dimensional Eulcr equations. To date most work has been done with the equivalent barotropic limit $\varepsilon=0$ and when the lower layer has infinite depth (see Kozlov 1983, 1985 for other examples). Stern (1985) and Pratt \& Stern (1986) study the evolution of large finite amplitude disturbances to a jump in potential vorticity of infinite length. Such a jump models the strong gradients that can occur in large-scale oceanic currents. They observe rapid distortion of the disturbance and the subsequent formation of lobe-like eddies that nearly detach from the main layer. Polvani et al (1989b) calculate V-state equilibria for $\Pi_{1}$ finite and $\Pi_{2}=0$, over a range of $\varepsilon$ and $\lambda$. In general these families are qualitatively similar to their Euler equivalents that correspond to $\lambda=0$. In two-vortex morger studics they find that filamentation is suppressed when $\varepsilon=0$ and $\lambda$ increases, but when $\varepsilon$ is small (and nonzero) filamentation occurs with subsequent merger like that found for the Euler equations. This is because the equivalent barotropic model is a singular limit of the two layer case inasmuch as (5) shows that the far field behavior of $G$ changes from the exponential decay of the $K_{0}$ Bessel function when $\varepsilon=0$ to logarithmic divergence when $\varepsilon$ is nonzero.

\section{FILAMENTATION}

Reference has been made to the phenomenon of filamentation whereby thin streams of vorticity are ejected, often repeatedly, from vortex patch boundaries and may form patterns of ever increasing complexity and apparently ever decreasing minimum spatial scale as they are convected and strained by the irrotational flow outside the vortex. Apart from its intrinsic mathematical interest this apparently ubiquitous property of contour dynamics may be of dynamical importance as a Lagrangian mixing mechanism for entrainment in real flows. Observations of analogous phenomenon in water bag computations of phase space hydrodynamics were noted by Berk et al (1970). Roberts \& Christiansen (1972) appear to have first remarked on filamentation of vortices in calculations of vortex merger while Deem \& Zabusky (1978) found extrusive filamentation following perturbations to circular vortex patches. Pullin (1981) observed intrusive contour filamentation when uniform wall-bounded vortex layers were subject to sufficiently large streamwise periodic perturbations. Here spikes of irrotational fluid intrude into the rotational layer. It was suggested that this phenomenon might account for turbulent bulge formation associated with inclined structures observed at the outer edge of turbulent boundary layers (e.g. Falco 1977). 
Dritschel (1988c) used a CS code to conduct a systematic study of the evolution of a circular patch subject to various perimeter initial perturbations, including the form

$$
r(\theta, 0)=R+\eta(\theta, t), \quad \eta(\theta, t)=\delta \exp \left[-1 / 2\left(\theta / \theta_{0}\right)^{2}\right],
$$

where $r$ is the perimeter at azimuthal angle $\theta, R$ is the unperturbed perimeter, $\delta=a R$ is the initial perturbation height, and $\theta_{0}=\pi / m$ is a measure of the bump width. Figure 3 shows an example with $a=0.05$ and $m=20$. The vorticity is $\omega=2 \pi$ below the contour and $\omega=0$ above. Filamentation first occurs near $t=24$, then repeating periodically to produce much fine structure. The results of many individual simulations suggested that filamentation occurred by a contour steepening mechanism
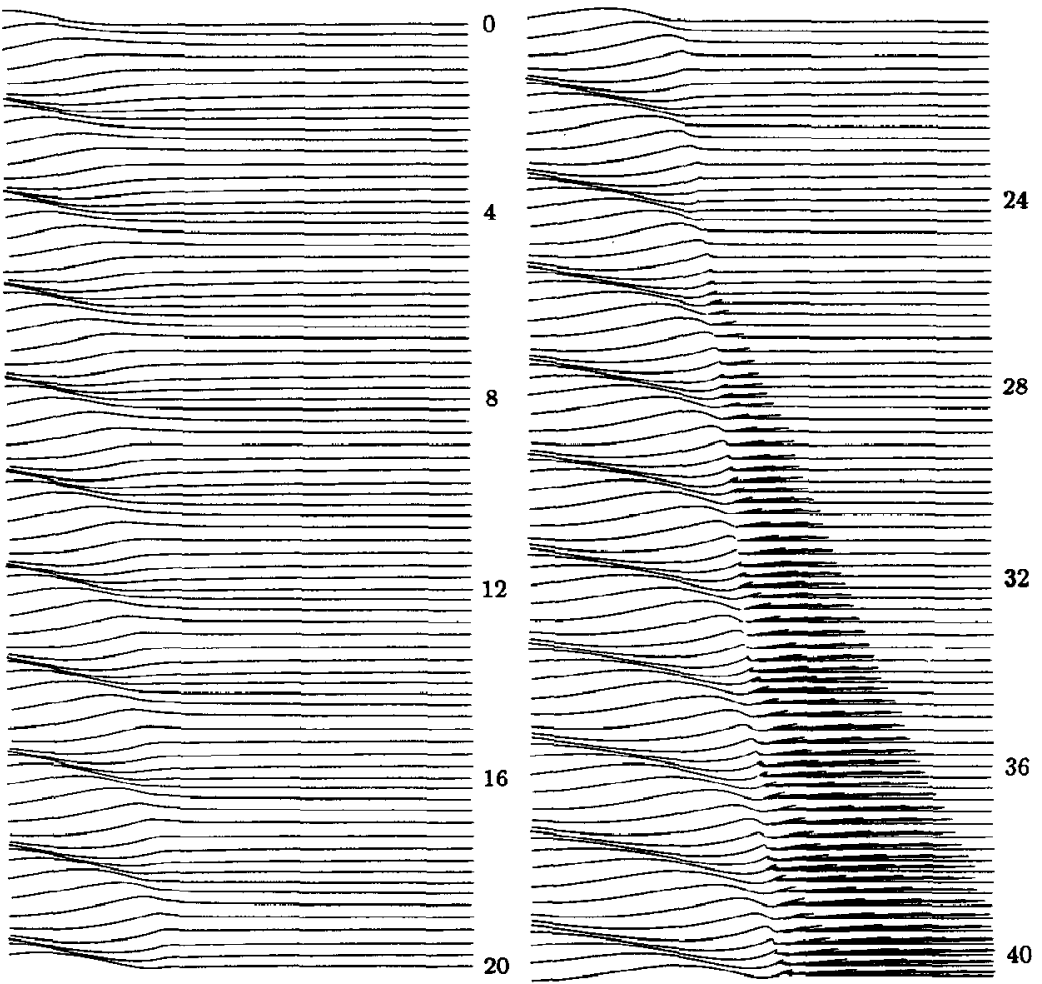

Figure 3 Evolution of a disturbance of the Equation (6) form on a circular vortex patch, $a=0.05, m=20$, for times $t$ as shown. $\eta(\theta, t)$ is plotted versus $\theta$ in $-\pi / 8<\theta<0$, at equally spaced intervals in $t$. [From Dritschel (1988c). Printed with permission.] 


\section{PULLIN}

in time $t_{\mathrm{f}}$ of order $a^{-2}$. Independent confirmation for $m=20$ has been given by Pullin \& Moore (1990) who have done further calculations (unpublished) that support the approximate empirical relation

$$
t_{\Gamma} \omega=\frac{2 \pi}{(a m)^{2}}\left(15+\frac{200}{m}\right)
$$

Dritschel (1988c) also obtained a third-order weakly nonlinear evolution equation governing perturbations to a circular vortex patch on a sphere. For the limiting planar case this reduces to

$$
\begin{aligned}
\eta_{\mathrm{t}} & +\frac{\omega}{2}[\eta+\mathscr{H}(\eta)]-\frac{\omega}{2 R^{2}} \eta \eta_{\theta} \\
& =\omega \frac{\partial}{\partial \theta}\left(-\frac{1}{3 R^{4}} \eta^{3}+\frac{1}{24 \pi R^{4}} \int_{0}^{2 \pi} \frac{\left[\eta(\theta, t)-\eta\left(\theta^{\prime}, t\right)\right]^{3}}{1-\cos \left(\theta-\theta^{\prime}\right)} d \theta^{\prime}\right)+O\left(\eta^{4}\right),
\end{aligned}
$$

where $\eta=\left(r^{2}-R^{2}\right) / 2$ and $\mathscr{H}$ is the Hilbert transform on the circle. On further expanding $\eta(\theta, t)$ in the two timescale form

$$
\eta(\theta, t)=a R \exp (\mathrm{i} \omega t / 2)\left[\bar{\eta}+A_{0}(\theta, \tau)+a A_{1}(\theta, t, \tau)+O\left(a^{2}\right)\right],
$$

where $\bar{\eta}$ is a mean value, an evolution equation for the envelope $A_{0}(\theta, \tau)$ is obtained in terms of the long timescale $\tau \equiv \omega t a^{2}$ in which the dimensionless perturbation height ( $a$ ) does not appear explicitly. Numerical solution of the envelope equation indicates secular behavior in $\tau$, which Dritschel identifies with the onset of filamentation in the fully nonlinear system. It is claimed that this provides evidence for the $a^{-2}$ scaling for $t_{\mathrm{f}}$ in the limit of vanishingly small $a$. Earlier Marsden \& Weinstein (1983) derived the form of (8) to second order, commenting that the dispersion operator $\mathscr{H}$ may be too weak to prevent phenomena like shock formation in the contour profile.

Pullin et al (1989) suggest several different paths to filamentation:

1. A strictly kinematic mechanism. This may operate whenever the initial contour shape is not near a patch equilibria. A heuristic explanation is that if, in an appropriate translating or corotating frame of reference hyperbolic critical points of the velocity field exist interior to the contour, then these will rapidly distort the vorticity and convect filaments away from the main core. This describes many examples of filamentation including that of Figure 1, the analogous phenomenon for magnetized electron plasmas (see Figure $6 b$ of Driscoll \& Fine 1990, who refer to a particle-wave resonance), filamentation following large amplitude perturbations to vortex layers (Pullin 1981, Stern \& Pratt 
1985, Grimshaw \& Yi 1991), and stable annular vortices (Dritschel 1986). It is not restricted to vortex patches (Melander et al 1987a).

2. Linear instability of vortex equilibria. This is similar to the first case except that linear instability provides growth in perturbation amplitude until the vorticity jump encounters a hyperbolic critical point that is initially exterior to the patch. Examples are the filamentation of the unstable Kirchoff ellipse with aspect ratio $a / b>3$ (Polvani et al 1989b) and the instability and filamentation of finite amplitude waves on vortex layers of finite thickness (Pullin et al 1989). The latter example may be interpreted as a weakly nonlinear instability if the undisturbed layer is viewed as the vortex equilibrium state.

3. The essentially nonlinear blocked cascade or shock formation mechanism found by Dritschel (1988c) in which nonlinear wave steepening occurs without growth in waveheight. Dritschel hypothesizes that almost all vortex patch equlibria will filament by this mechanism when perturbed by an isolated disturbance of arbitrarily small waveheight. Evidence that this occurs for perturbed continuous distributions is provided by a demonstration of filamentation on a four-layer model of the patch cdge.

\section{Corner Formation}

It is known that solution branches for many families of vortex equilibria terminate where the contour shape forms a $90^{\circ}$ corner (Saffman \& Szeto 1980,1981 , Wu et al 1984, Broadbent \& Moore 1985, Overman 1986), examples being V-states with $m \geq 3$ (Deem \& Zabusky 1978) and waves of finite amplitude on wall-bounded vortex layers (Broadbent \& Moore 1985). Overman (1986) shows that equilibria with a cusped contour are admissible as possible steady solutions of the Euler equations but there are no known cases. Let us use polar coordinates $(r, \theta)$ with origin at the corner and measure $\theta$ counterclockwise from the negative $y$-axis (an axis of symmetry). Let the uniform vorticity in the upper fluid $\omega_{1}$ and that in the lower fluid be $\omega_{2}<\omega_{1}$. Then in $\pi \geq \theta \geq 0$ the local solution for the stream functions $\psi_{1}$ and $\psi_{2}$ describing steady flow with a contour corner in $\pi / 2 \geq \theta \geq-\pi / 2$ at the origin is

$$
\begin{aligned}
\psi_{1}= & \frac{\omega_{1}-\omega_{2}}{4 \pi}\left[r^{2} \log (r) \cos (2 \theta)-\theta r^{2} \sin (2 \theta)\right] \\
& -\frac{\omega_{1} r^{2}}{4}+\frac{\omega_{1}-\omega_{2}}{4} r^{2} \sin 2 \theta+O\left(r^{4}\right), \\
\psi_{2}= & \frac{\omega_{1}-\omega_{2}}{4 \pi}\left[r^{2} \log (r) \cos (2 \theta)-\theta r^{2} \sin (2 \theta)\right]-\frac{\omega_{2} r^{2}}{4}+O\left(r^{4}\right) .
\end{aligned}
$$


To leading order the contour in $\pi \geq \theta \geq 0$ has the shape

$$
\theta=\frac{\pi}{4}-\frac{\pi\left(3 \omega_{2}+\omega_{1}\right)}{8\left(\omega_{1}-\omega_{2}\right) \log (r)}+O\left(\frac{1}{\log (r)}\right)^{2}
$$

The velocity field is continuous but nonanalytic at the corner where strain rates are unbounded.

Is spontaneous corner or other singularity formation in a finite time possible for unsteady contour evolution? (See note added in proof.) Such events are not ruled out by global uniqueness theorems for the twodimensional Euler equations (Yudovitch 1963), although according to Baker \& Shelley (1990) it can be shown that vortex jumps will remain smooth for small times. Buttke (1989) reported the formation of a tangent slope discontinuity in a two-vortex merger calculation. Dritschel \& McIntyre (1990) calculate the same flow with high resolution CD and find smooth contour evolution. They attribute Buttke's result to spurious infinite strain rates produced by the use of a contour representation with $90^{\circ}$ corners. Pullin \& Moore (1990) speculate that some instances of filamentation may result from incipient corner formation unresolved by finite precision CD-but their results do not support this idea. Spectral methods used for the numerical tracking of singularities in evolution problems may provide decisive evidence of corner or cusp formation, although their application to $\mathrm{CD}$ has not yet been reported. A related question is the stability of steady vortex equilibria with corners. The linear stability analysis is complicated by two factors. First, it requires highly accurate numerical results for the limiting equilibrium statc. Secondly, a continuous spectrum [as is found for an axisymmetric analog of CD in the Hills spherical vortex (Moffatt \& Moore 1978)] is likely to exist.

\section{Contour Dynamics as a Hamiltonian System}

Answers to delicate questions concerning possible singularity formation and the onset of fine scales in CD may benefit from a Hamiltonian approach. Marsden \& Weinstein (1983) discuss the geometric Hamiltonian structure possessed by the incompressible Euler equations (see also Herbert 1983 and Salmon 1988 for a review) when viewed on a surface known as a coadjoint orbit, which is a symplectic submanifold consisting of all possible points accessible from the initial conditions. However, the problem of determining a set of canonical coordinates and conjugate momenta is nontrivial because coadjoint orbits are not flat but are generally infinite dimensional abstract objects. An exception is a system of $N$ point vortices in which the coadjoint orbit has the required properties, the phase space is $R^{N}$, and the canonical coordinates are just the $x$ and $y$ 
vortex positions. For vortex patches Rouhi (personal communication) showed that while the patch shape $r(\theta, t)$ remains single-valued in $\theta$, the coefficients $a_{i}(t)$ and $b_{i}(t)$ in the Fourier series for $r(\theta, t)$ are a set of local canonical coordinates. When contour overturning occurs the patch breaks out of the locally flat region of the coadjoint orbit and global canonical coordinates cannot be found.

Generally, Hamiltonian systems have the property that any functional $F(\omega)$ obeys an equation of the Lie-Poisson type

$$
\frac{d F}{d t}=\{F, H\}
$$

where $\{.,$.$\} is the Poisson bracket and H$ is the Hamiltonian function, which for vortex patches is the excess energy and is given by

$$
H=\frac{1}{16 \pi} \sum_{j} \sum_{k} \Delta \omega_{j} \Delta \omega_{k} \oint_{\mathscr{G}_{j}} \oint_{\mathscr{G}_{k}}\left(\mathbf{r}_{j}^{\prime}-\mathbf{r}_{k}\right)^{2} \log \left|\mathbf{r}_{j}^{\prime}-\mathbf{r}_{k}\right| d \mathbf{r}_{j}^{\prime} d \mathbf{r}_{k} .
$$

Numerical integrators that preserve symplectic structure (in the sense of preserving to machine precision the Poincare invariants) are apparently well suited to the numerical study of explicit Hamiltonian systems; see review by Channel \& Scovel 1990 and also Pullin \& Saffman 1990 for an application to the long time integration of four-vortex motion. Ge \& Marsden (1988) and Scovel (1990) discuss the construction of Lie-Poisson integrators for systems like (13) when only the Poisson bracket is known. The application of such methods to $\mathrm{CD}$ remains an area for future research.

\section{CONCLUDING REMARKS}

Driven by tractable mathematics and manageable numerics, contour dynamics methods have undoubtedly contributed to our understanding the dual phenomena of enstrophy cascade to fine scales and the movement of energy to larger scales for two-dimensional vortex dynamics in the limit of infinite Reynolds numbers. Many open problems remain including the validity of the axisymmetrization principle and related questions of long time statistical equilibria, the relevance of filamentation to flows with continuous vorticity, and the possibility of singularity formation in contour shapes. Saffman (1990) suggests that the role of vortex dynamics in elucidating our understanding of turbulence has been primarily of qualitative rather than quantitative significance. While there have been many examples of contour dynamics calculations that resemble natural and laboratory fluid flows, some of which are discussed in this article, explicit 
incorporation of ideas flowing from these numerical experiments into models capable of quantiative prediction remains to be demonstrated.

\section{ACKNOWLEDGMENTS}

The author wishes to thank Drs. G. R. Baker, D. I. Meiron, D. W. Moore, and M. J. Shelley for helpful discussions. Thanks are due especially to Dr. P. G. Saffman for providing valuable comments on a manuscript draft and to Dr. A. Rouhi for explaining the concepts of Hamiltonian dynamics as applied to vortex patches.

\section{Literature Cited}

Acton, E. 1976. A modelling of large eddies in an axisymmetric jet. J. Fluid Mech. 98 : $1-31$

Aref, H. 1983. Integrable, chaotic, and turbulent vortex motion in two-dimensional flows. Annu. Rev. Fluid Mech. 15: 345-89

Aref, H., Siggia, E. D. 1980 . Vortex dynamics of the two-dimensional turbulent shear layer. J. Fluid Mech. 100: 705-37

Aref, H., Siggia, E. D. 1981. Evolution and breakdown of a vortex street in two dimensions. J. Fluid Mech. 109: 435-63

Baker, G. R. 1990. A study of the numerical stability of contour dynamics. Philos. Trans. $R$. Soc. London Ser. A 333: 391-400

Baker, G. R., Shelley, M. J. 1990. On the connection between thin vortex layers and vortcx shccts. J. Fluid Mech. 15: 161-94

Batchelor, G. K. 1967. An introduction to Fluid Dynamics. Cambridge: Cambridge University Press

Berk, H. L., Neilsen, C. E., Roberts, K. V. 1970. Phase space hydrodynamics of equivalent nonlinear systems. Phys. Fluids 13 : 980-95

Berk, H. L., Roberts, K. V. 1970. The "water bag" model. Method Comput. Phys. 9: 8897

Broadbent, E. G., Moore, D. W. 1985. Waves of extreme form on a layer of uniform vorticity. Phys. Fluids 28: 1561-63

Buttke, T. F. 1989. The observation of singularities in the boundary of patches of constant vorticity. Phys. Fluids A I: 1283-88

Caflisch. R. E. 1988. Mathematical analysis of vortex dynamics. In Mathematical Aspects of Vortex Dynamics, ed. R. E. Caflisch. pp. 1-24. Philidelphia: Soc. Ind. Appl. Math.

Campbell, L. J., Ziff, R. M. 1978. A catalogue of two-dimensional vortex patterns. Los. Alamos Rep. LA-7384-MS. 40 pp.

Campbell, L. J., Ziff, R. M. 1979. Vortex patterns and energies in a rotating superfluid. Phys. Rev. B 20: 1886-1902

Cantwell, B. 1989. Future dircetions in turbulence research and the role of organized motion. In Whither Turbulence, ed. J. Lumley, pp. 97-131. New York: Springer Verlag

Channel, P. J. \& Scovell, J. C. 1990 . Symplectic integration of Hamiltonian systems. Nonlinearity 3: 231-59

Chorin, A. J. 1980. Vortex models and boundary layer instability. SIAMJ.Sci. Stat. Comput. 1: 1-21

Christiansen, J. P., Zabusky, N. J. 1973. Instability, coalescence and fission of finite-area vortex structures. J. Fluid Mech. 61: 21943

Corcos, G. M., Sherman, F. S. 1984. The mixing layer: deterministic models of a turbulent flow. Part 1. Introduction and the two-dimensional flow. J. Fluid Mech. 139: 29-65

Couder, Y., Basdevant, C. 1986. Experimental and numerical study of vortex couples in two-dimensional flows. J. Fluid Mech. 173: 225-51

Deem, G. S., Zabusky, N. J. 1978. Vortex waves: Stationary "V states," interactions, recurrence and breaking. Phys. Rev. Lett. 40: 859-62

dePackh, C. D. 1962. The water bag method model of a sheet electron beam. $J$. Electron. Control. 13: 417-24

Driscoll, C. F., Fine, K. S. 1990. Experiments on vortex dynamics in pure electron plasmas. Phys. Fluids B 2: 1359-66

Dritschel, D. G. 1985. The stability and energetics of corotating uniform vortices. $J$. Fluid Mech. 157: 95134

Dritschel, D. G. 1986. The non-linear evolution of rotating configurations of uniform vorticity. J. Fluid Mech. 172: 157-82

Dritschel, D. G. 1988a. Nonlinear stability 
bounds for inviscid, two-dimensional, parallel or circular fows with monotonic vorticity, and the analogous three-dimensional quasi-geostrophic flows. J. Fluid Mech. 191: 575-82

Dritschel, D. G. 1988b. Contour surgery: A topological reconnection scheme for extended interactions using contour dynamics. J. Comput. Phys. 77: 240-66

Dritschel, D. G. 1988c. The repeated filamentation of two-dimensional vorticity interfaces. J. Fluid Mech. 194: 511-47

Dritschel, D. G. 1989. Contour dynamics and contour surgery: Numerical algorithms for extended high-resolution modelling of vortex dynamics in two-dimensional, inviscid, incompressible flows. Comput. Phys. Rep. 10: 79-146

Dritschel, D. G. 1990a. On the stabilization of a two-dimensional vortex strip by adverse shear. J. Fluid Mech. 206: 193221

Dritschel, D. G. 1990b. The stability of elliptical vortices in an external straining flow. J. Fluid Mech. 210: 223-61

Dritschel, D. G., McIntyre, M. E. 1990. Does contour dynamics go singular? Phys. Fluids A 2: 748-53

Falco, R. E. 1977. Coherent structures in the outer region of a turbulent boundary layer. Phys. Fluids Suppl. 20: 124-32

Fornberg, B. 1977. A numerical study of 2D turbulcnce. J. Comput. Phys. 25: 1-31

Fraenkel, L. E. 1972. Examples of steady vortex rings of small cross-section in an ideal fluid. J. Fluid Mech. 51: 119-35

Ge, Z., Marsden, J. E. 1988. Lie-Poisson Hamiltonian-Jacobi theory and Lie-Poisson integrators. Phys. Rev. Lett. A 133: 134-39

Grimshaw, R. \& Yi, Z. 1990. Evolution of a potential vorticity front over a topographic slope. J. Phys. Oceanogr. 21: 1240-55

Henshaw, W. D., Kreiss, H. O., Reyna, L. G. 1989. On the smallest scale for the incompressible Navier-Stokes equations. Theor. Comput. Fluid Dyn. 1: 65-95

Herbert, D. J. 1983. A HamiltonianLagrangian system of vortex boundary curves. J. Math. Phys. 24: 1672-78

Hogg, N., Stommel, H. 1985. The heton, an elementary interaction between discrete baroclinic geostrophic vortices and its implications concerning eddy heat-flow. Proc. R. Soc. London A 397: 1-20

Hussaini, M. Y., Zang, T. A. 1987. Spectral methods in fluid dynamics. Annu. Rev. Fluid Mech. 19: 339-67

Jacobs, P. A., Pullin, D. I. 1985. Coalescence of stretching vortices. Phys. Fluids 28: 1619-25

Jacobs, P. A., Pullin, D. I. 1989. Multiple- contour-dynamic simulation of eddy scales in the plane shear layer. $J$. Fluid Mech. 199: 89-124

Kamm, J. R. 1987. Shape and stability of two-dimensional uniform vorticity regions. PhD thesis, Calif. Inst. Tech.

Kida, S. 1981. Motion of an elliptic vortex in a uniform shear flow. J. Phys. Soc. Jpn. 50: $3517-20$

Koochesfahani, M. M., Dimotakis, P. E. 1986. Mixing and chemical reactions in a turbulent liquid mixing layer. J. Fluid Mech. 170: 83-112

Kozlov, V. F. 1983. The method of contour dynamics in model problems of the ocean topographysical cyclogenesis. Izv. Atmos. Ocean Phys. 19, 635-640

Kozlov, V. F. 1985. Construction of a numerical model of geostrophic eddies in a barotropic fluid based on the contour dynamics method. Izv. Atmos. Ocean Phys. 21: 161-63

Krasny, R. 1986. Desingularization of vortex sheet rollup. J. Comput. Phys. 65: 292-313

Lamb, H. 1932. Hydrodyamics. New York: Dover

Legras, B., Dritschel, D. G. 1990. Vortex stripping. Preprint

Leonard, A. 1980. Vortex methods for flow simulation. J. Comput. Phys. 37: 289-335

Leonard, A. 1985. Computing three-dimensional incompressible flows with vortex elements. Annu. Rev. Fluid Mech. 17: 52359

Levy, R. H., Hockney, R. W. 1968. Computer experiments on low-density crossedfield electron beams. Phys. Fluids 11: 77671

Lin, S. J., Corcos, G. M. 1984. The mixing layer: deterministic models of a turbulent flow. Part 3. The effect of plane strain on the dynamics of streamwise vortices. $J$. Fluid Mech. 141: 139-78

Lundgren, T. S. 1982. Strained spiral vortex model for turbulent fine structure. Phys. Fluids 25: 2193-2203

Marsden, J., Weinstein, A. 1983. Coadjoint orbits, vortices, and Clebsch variables for incompressible fluids. 1983 Physica $7 D$ $305-23$

McWilliams, J. C. 1984. The emergence of isolated coherent vortices in turbulent flow. J. Fluid Mech. 146: 21-43

Meiron, D. I., Baker, G. R., Orszag, S. A 1982. Analytic structure of vortex sheet dynamics I: Kelvin-Helmholtz instability. J. Fluid Mech. 114: 283-98

Meiron, D. I., Saffman, P. G., Schatzman, J. C. 1984. The linear two-dimensional stability of inviscid vortex streets of finite cored vortices. J. Fluid Mech. 147: 187 212

Melander, M. V., McWilliams, J. C., Zabu- 


\section{PULLIN}

sky, N. J. 1987a. Axisymmetrization and vorticity gradient intensification of an isolated two-dimensional vortex through filamentation. J. Fluid Mech. 178: 13759

Melander, M. V., Overman, E. A. II, Zabusky, N. J. 1987b. Computational vortex dynamics in two and three dimensions. Appl. Num. Math. 3: 59-80

Melander, M. V., Zabusky, N. J., McWilliams, J. C. 1988. Symmetric vortex merger in two dimensions: Causes and conditions. J. Fluid Mech. 195: 303-40

Melander, M. V., Zabusky, N. J., Styczek, A. S. 1986. A moment model for vortex interactions of the two-dimensional Euler equations. Part 1 . Computational validation of a Hamiltonian elliptical representation. J. Fluid Mech. 167: 95-115

Miller, J. 1990. Statistical mechanics of Euler equations in two dimensions. Phys. Rev. Lett. 65: 2137-40

Moffatt, H. K., Moore, D. W. 1978. The response of Hill's spherical vortex to a small axisymmetric disturbance. $J$. Fluid Mech. 87: 749-60

Moore, D. W. 1974. A numerical study of the roll-up of a finite vortex sheet. J. Fluid Mech. 63: 225-35

Moore, D. W. 1978. The equation of motion of a vortex layer of small thickness. Stud. Appl. Math. 58: 119-40

Moore, D. W. 1979. The spontaneous appearance of a singularity in the shape of an evolving vortex sheet. Proc. $R$. Soc. London A 365: 105-19

Moore, D. W. 1983. On the point vortex method. SIAM J. Sci. Stat. Comput. 2: 65-84

Moore, D. W. 1985. Numerical and analytical aspects of Helmholtz instability. In Theoretical \& Applied Mechanics, ed. F. 1. Niordson, N. Hoff, pp. 263-74. Amsterdam: N. Holland

Moore, D. W., Saffman, P. G. 1971. Structure of a line vortex in an imposed strain. In Aircraft Turbulence and Wakes, ed. J. H. Olsen, A. Goldburg, M. Rogers, pp. 339-54. New York: Plenum

Neu, J. C. 1984. The dynamics of a columnar vortex in an imposed strain. Phys. Fluids 27: 2397-2402

Norbury, J. 1972. A steady vortex ring close to Hill's spherical vortex. J. Fluid Mech. 72: $253-84$

Norbury, J. 1973. A family of steady vortex rings. J. Fluid Mech. 57: 417-31

Overman, E. A. II, 1986. Steady state solutions of the Euler equations in two-dimensions. II. Local analysis of limiting Vstates. SIAM J. Appl. Math. 46: 765-800

Overman, E. A. II, Zabusky, N. J. 1982. Coaxial scattering of Euler-equation translating $\mathrm{V}$-states via contour dynamics. J. Fluid Mech. 125: 187-202

Overman, E. A. II, Zabusky, N. J. 1983. Evolution and merger of isolated vortex structures. Phys. Fluids 25: 1297-1305

Pedlosky, J. 1987. Geophysical Fluid Dynamics. New York: Springer

Pierrehumbert, R. T. 1980. A family of steady, translating vortex pairs with distributed vorticity. J. Fluid Mech. 99: 12944

Pierrehumbert, R. T. 1981. Corrigendum, A family of steady, translating vortex pairs with distributed vorticity. J. Fluid Mech 102: 478

Pointin, Y. B., Lundgren, T. S. 1976. Statistical mechanics of two-dimensional vortices in a bounded container. Phys. Fluids 10: 1459-70

Polvani, L. M., Carton, X. J. 1990 . The tripole: a new coherent vortex structure of incompressible two-dimensional flows. Geophys. Astro. 51: 87-102

Polvani, L. M., Flierl, G. R. 1986. Generalized Kirchoff Vortices. Phys. Fluids 29: 2376-97

Polvani, L. M., Flierl, G. R., Zabusky, N. J. 1989a. Filamentation of unstable vortex structures via separatrix crossing: A quantitative estimate of onset time. Phys. Fluids A 1: $181-84$

Polvani, L. M., Zabusky, N. J., Flierl, G. R. 1989b. Two-layer geostrephic vortex dynamics. Part 1. Upper-layer V-states and merger. J. Fluid Mech. 205: 215-42

Pozrikidis, C. 1986. The non-linear instability of Hill's vortex. J. Fluid Mech. 168: 337-67

Pozrikidis, C., Higdon, J. J. L. 1985. Nonlinear Kelvin-Helmholtz instability of a finite layer. J. Fluid Mech. 157: 225-63

Pozrikidis, C., Higdon, J. J. L. 1987. Instability of compound layers and wakes. Phys. Fluids 30: 2965-75

Pratt, L. J., Stern, M. E. 1986. Dynamics of potential vorticity fronts and eddy detachment. J. Phys. Oceangr. 16: 1101-20

Pullin, D. I. 1981. The nonlinear behaviour of a constant vorticity layer at a wall. $J$. Fluid Mech. 108: 401-21

Pullin, D. I., Jacobs, P. A. 1986. Inviscid evolution of stretched vortex arrays. $J$. Fluid Mech: 171: 377-406

Pullin, D. I., Jacobs, P. A., Grimshaw, R. H. J., Saffman, P. G. 1989. Instability and filamentation of finite-amplitude waves on vortex layers of finite thickness. $J$. Fluid Mech. 209: 359-85

Pullin, D. I., Moore, D. W. 1990. Remark on a result of D. Dritschel. Phys. Fluids $A$ 2. 1039-41

Pullin, D. I., Saffman, P. G. 1990. Longtime symplectic integration of four-vortex 
motion. Proc. R. Soc. London A 432: 481-94

Rayleigh, Lord 1880 . On the stability, or instability, of certain fluid motions. Proc. London Math. Soc. XI: 57-70. Sci. Papers 1: 474-87. New York: Dover

Roberts, K. V., Christiansen, J. P. 1972. Topics in computational fluid mechanics. Computer Phys. Commun. Suppl. 3: 14-32

Rosenhead, L. 1931. The formation of vortices from a surface of discontinuity. Proc. R. Soc. London Ser. A 134: 170-92

Sadovskii, V. S. 1971. Vortex regions in a potential stream with a jump of Bernoulli's constant at the boundary. Prikl. Mat. Mekh. 35: 773-79

Saffman, P. G. 1981. Dynamics of vorticity. J. Fluid Mech. 106: 49-58

Saffman, P. G. 1982. Structurc and stability of streets of finite vortices. In Vortex Motion, ed. H. G. Hornung, E.-A. Müller, pp. 142-153. Braunschweig/Wiesbaden: Friedr. Vieweg. \& Sohn

Saffman, P. G. 1985. Perspective in vortex dynamics. In Perspective in Fluid Mechanics, ed. D. Coles, pp. 91-102. New York: Springer-Verlag

Saffman, P. G. 1988. The stability of vortex arrays to two- and three-dimensional disturbances. Fluid Dyn. Res. 3: 13-31

Saffman, P. G. 1990. Vortex dynamics and turbulence. In Proc. Nato Advanced workshop on The Global Geometry of Turbulence: Impact of nonlinear dynamics, Rota Spain, 8-14 July

Saffman, P. G., Baker, G. R. 1979. Vortex interactions. Annu. Rev. Fluid Mech. 11: $95-122$

Saffman, P. G., Schatzman, J. C. 1981. Properties of a vortex street of finite vortices. SIAM J. Sci. Stat. Comput. 2: 285-95

Saffman, P. G., Schatzman, J. C. 1982. Stability of a vortex street of finite vortices. $J$. Fluid Mech. 117: 171-85

Saffman, P. G., Szeto, R. 1980. Equilibrium shapes of a pair of equal uniform vortices. Phys. Fluids 23: 2339-42

Saffman, P. G., Szeto, R. 1981. Structure of a linear array of uniform vortices. Stud. Appl. Math. 65: 223-48

Salmon, R. 1988. Hamiltonian fluid mechanics. Annu. Rev. Fluid Mech. 20: 225-56
Scovel, C. 1990. Symplectic integration of Hamiltonian systems. Preprint

Shariff, K. Leonard, A., Ferziger, J. H. 1989. Dynamics of a class of vortex rings. NASA Tech. Mem. 102257. 268 pp.

Smith, R. A., Rosenbluth, M. N. 1990. Algebraic instability of hollow electron columns and cylindrical vortices. Phys. Rev. Lett. 64: 649-52

Stern, M. E. 1985. Large scale wave breaking and shingle formation. J. Phys. Oceanogr. 15: $1274-83$

Stern, M. E., Pratt, L. J. 1985. Dynamics of vorticity fronts. J. Fluid Mech. 161: 513-32

$\mathrm{Su}$, C. H. 1979. Motion of fluid with constant vorticity in a singly connected region. Phys. Fluids 22: 2032 33

Westwater, F. L. 1935. Rolling up of the surface of discontinuity behind an aerofoil of finite span. Aerosp. Res. Comm. Rep. Memo. No. 1692

Winant, C. D., Browand, F. K. 1974. Vortex pairing: The mechanism of turbulent mixing-layer growth at moderate Reynolds number. J. Fluid Mech. 63: 237-55

Wu, H. M., Overman, E. A. II, Zabusky, N. J. 1984. Steady-state solutions of the Euler equations in two dimensions: Rotating and translating $V$-states with limiting cases. Numerical algorithms and results. J. Comput. Phys. 53: 42-71

Yudovitch, V. I, 1963. Non stationary flow of an ideal incompressible liquid. $Z h$. Vych. Mater. 3: 1032

Zabusky, N. J. 1981. Computational synergetics and mathematical innovation. $J$. Comput. Phys. 43: 195-249

Zabusky, N. J., Hughes, M. H., Roberts, K. V. 1979. Contour dynamics for the Euler equations in two dimensions. $J$. Comput. Phys. 30: 96-106

Zabusky, N. J., Overman, E. A. II 1983. Regularization of contour dynamical algorithms. I. Tangential regularization. J. Comput. Phys. 52: 351-73

Zou, Q., Overman, E. A. II, Wu, H.-M., Zabusky, N. J. 1988. Contour dynamics for the Euler equations: Curvature controlled initial node placement and accuracy. J. Comput. Phys. 78: 350-68

\section{NOTE ADDED IN PROOF}

This issuc appcars to have been settled by Jean-Yves Chemin (1991, Existence globalle pour le problème des poches de tourbillon, CR Acad. Sci. Paris Ser. I, 803-6) who has reported a proof showing that if the contour is initially in $C^{k+\varepsilon}$ for some positive integer $k$ and some positive $\varepsilon$, then it remains in $C^{k+\varepsilon}$ for all time. 
A Annual Review of Fluid Mechanics

Volume 24, 1992

\section{CONTENTS}

Gröbli's Solution of THE Three-Vortex Problem, Hassan Aref, Nicholas Rott, and Hans Thomann 1

Modeling of Two-Phase Slug Flow, J. Fabre and A. Liné 21

Measuring the Flow Properties of Yield Stress Fluids, Q. D. Nguyen and D. V. Boger

Contour Dynamics Methods, D. I. Pullin

Parabolized/Reduced Navier-Stokes Computational TeChNiques, Stanley G. Rubin and John C. Tannehill

Topological Methods in Hydrodynamics, $V$. I. Arnold and B. A. Khesin

Finite Element Methods for Navier-Stokes Equations, Roland Glowinski and Olivier Pironneau

ATMOSPHERIC TURBulenCE, John C. Wyngaard 205

Vortex RINGS, Karim Shariff and Anthony Leonard

Helicity in Laminar and Turbulent Flow, $H . K$. Moffatt and A. Tsinober

Hydrodynamic Phenomena in Suspensions of Swimming Microorganisms, T. J. Pedley and J. O. Kessler

Numerical Models of Mantle Convection, G. Schubert

Wavelet Transforms and Their Applications to Turbulence, Marie Farge

Dynamo Theory, P. H. Roberts and A. M. Soward

INDEXES

Subject Index

Cumulative Index of Contributing Authors, Volumes 1-24

526

Cumulative Index of Chapter Titles, Volumes 1-24

530 